\title{
Correction to: Bioactive Factors and Processing Technology for Cereal Foods
}

Jing Wang, Baoguo Sun, and Rong Tsao

\section{Correction to:}

J. Wang et al. (eds.), Bioactive Factors

and Processing Technology for Cereal Foods, https://doi.org/10.1007/978-981-13-6167-8

The book was inadvertently published with an incorrect spelling of the Author's name and this was updated globally in the book as RongTsao Cao whereas it should be Rong Tsao. 